\title{
On Properties of Holomorphic Functions in Quaternionic Analysis
}

\author{
Michael Parfenov ${ }^{*}$ \\ Bashkortostan Branch of Russian Academy of Engineering, Ufa, Russia \\ *Corresponding author: parfenov.48@bk.ru
}

\begin{abstract}
We draw the conclusions from the earlier presented quaternionic generalization of Cauchy-Riemann's equations. The general expressions for constituents of $\mathbb{H}$-holomorphic functions as well as the relations between them are deduced. The symmetry properties of constituents of $\mathbb{H}$-holomorphic functions and their derivatives of all orders are proved. For full derivatives it is a consequence of uniting the left and right derivatives within the framework of the developed theory. Some $\mathbb{H}$-holomorphic generalizations of $\mathbb{C}-$ holomorphic functions are discussed in detail to demonstrate particularities of constructing H-holomorphic functions. The power functions are considered in detail.
\end{abstract}

Keywords: quaternionic holomorphic functions, quaternionic analysis, quaternionic generalization of CauchyRiemann's equations, functions of hypercomplex variables

Cite This Article: Michael Parfenov, "On Properties of Holomorphic Functions in Quaternionic Analysis." American Journal of Mathematical Analysis, vol. 5, no. 1 (2017): 17-24. doi: 10.12691/ajma-5-1-4.

\section{Introduction}

There exist various versions of quaternionic analysis. They imply, explicitly or implicitly, the "left"and "right" definitions of a quaternionic derivative (e. g., $[1-6,8,9,12,13]$ ), which are regarded as equivalent. Each of them by itself is incomplete (may be called "inessentially adequate"), since only the left or right version considered separately doesn't represent all arbitrary rotations of vectors in space [7,11] by the underlying algebra.

Moreover, they don't enable to construct quaternionic holomorphic functions from complex holomorphic counterparts by direct replacing a complex variable by a quaternionic in expressions for complex holomorphic functions, whilst a similar way exists in complex analysis. We develop further the "essentially adequate" theory [11] that unites both approaches and solves this problem.

We denote a quaternionic variable by $p=x+y i+$ $z j+u k=a+b j \in \mathbb{H}$, where $a=x+y i, b=z+u i$ $(x, y, z, u$ are real values, $i, j, k$ are quaternionic basis vectors, $\mathbb{H}$ denotes a quaternionic space), and a quaternion-valued (briefly, quaternionic) function by $\psi(p)=\phi_{1}+\phi_{2} j$, where $\phi_{1}=\phi_{1}(a, b)=\psi_{1}+\psi_{2} i$ and $\phi_{2}=\phi_{2}(a, b)=\psi_{3}+\psi_{4} i\left(\psi_{1}, \psi_{2}, \psi_{3}, \psi_{4}\right.$ are real-valued functions of $x, y, z, u)$. In accordance with [11] we define a $\mathbb{H}$ - holomorphic function as follows.

Definition 1.1. It is assumed that the constituents $\phi_{1}(a, b)$ and $\phi_{2}(a, b)$ of a quaternionic function $\psi(p)=$ $\psi(a, b)=\phi_{1}+\phi_{2} j$ possess continuous first-order partial derivatives with respect to $a, \bar{a}, b$, and $\bar{b}$ in some open connected neighborhood $G_{4} \in \mathbb{H}$ of a point $p \in G_{4}$. Then a function $\psi(p)$ is said to be $\mathbb{H}$-holomorphic and denoted by $\psi_{H}(p)$ at a point $p$, if and only if the functions $\phi_{1}(a, b)$ and $\phi_{2}(a, b)$ satisfy in $G_{4}$ the following quaternionic generalization of Cauchy-Riemann's equations:

$$
\left\{\begin{array}{l}
1)\left(\partial_{a} \phi_{1} \mid=\left(\partial_{\bar{b}} \bar{\phi}_{2} \mid, 2\right)\left(\partial_{a} \phi_{2} \mid=-\left(\partial_{\bar{b}} \bar{\phi}_{1} \mid,\right.\right.\right. \\
3)\left(\partial_{a} \phi_{1} \mid=\left(\partial_{b} \phi_{2} \mid, 4\right)\left(\partial_{\bar{a}} \phi_{2} \mid=-\left(\partial_{\bar{b}} \phi_{1} \mid .\right.\right.\right.
\end{array}\right.
$$

Here and in the sequel, the complex conjugation is denoted as usual (for example, $\bar{\phi}_{2}$ ); the brackets (.. with the closing vertical bar indicate that the transition $a=\bar{a}=x$ has been already performed in expressions enclosed in brackets.

Thus, the $\mathbb{H}$-holomorphicity condition is defined so that during the check of quaternionic holomorphicity of any quaternionic function we have to do the transition $a=\bar{a}=x$ in already computed expressions for partial derivatives of functions $\phi_{1}$ and $\phi_{2}$ to be used in the equations (1). However, this does not mean that we deal with triplets in general; this transition cannot be initially done for quaternionic variables and functions [11]. Any quaternionic function of a quaternion variable remains the same 4-dimensional quaternionic function regardless of whether we check its quaternionic holomorphicity or not.

Equations (1-1,2) and (1-3,4) represent left and right $\mathbb{H I}$-holomorphicity conditions, respectively. The additional condition $a=\bar{a}=x$, reflecting the requirement of an uniqueness of a derivative in space, unites them and allows us to construct $\mathbb{H}$-holomorphic functions from the complex holomorphic (briefly, $\mathbb{C}$-holomorphic) counterparts by replacing variables, as noted above. We will establish some properties of $\mathbb{H}$-holomorphic functions, defined by equations (1).

The purpose of this article is to obtain the general expressions for constituents $\phi_{1}(a, b)$ and $\phi_{2}(a, b)$ of $\mathbb{H}-$ holomorphic functions as well as some important relations between them. 
Denote a $k^{\prime}$ th derivative of a $\mathbb{H}$-holomorphic function $\psi_{H}(p)$ by

$$
\psi_{H}^{(k)}(p)=\phi_{1}^{(k)}+\phi_{2}^{(k)} j,
$$

where the constituents $\phi_{1}^{(k)}$ and $\phi_{2}^{(k)}$ are expressed [11] by

$$
\begin{aligned}
& \phi_{1}^{(k)}=\partial_{a} \phi_{1}^{(k-1)}+\partial_{\bar{a}} \phi_{1}^{(k-1)}, \\
& \phi_{2}^{(k)}=\partial_{a} \phi_{2}^{(k-1)}+\partial_{\bar{a}} \phi_{2}^{(k-1)} ;
\end{aligned}
$$

$\phi_{1}^{(k-1)}$ and $\phi_{2}^{(k-1)}$ are the constituents of the $(k-1)^{\prime}$ th derivative of $\psi_{H}(p)$, represented in the Cayley-Dickson doubling form as $\psi(p)^{(k-1)}=\phi_{1}^{(k-1)}+\phi_{2}^{(k-1)} j, k \geq 1$; $\phi_{1}^{(0)}=\phi_{1}(a, b)$ and $\phi_{2}^{(0)}=\phi_{2}(a, b), k=1$. As we will see later, these formulae generalize expressions for derivatives of $\mathbb{C}$-holomorphic functions.

To represent all expressions in the Cayley-Dickson doubling form [6], which plays a principal role further, we use the identity

$$
j z=\bar{z} j \text { for any } z \in \mathbb{C} .
$$

\section{Expressions for Constituents $\phi_{1}$ and $\phi_{2}$}

When formulating the essentially adequate quaternionic generalization of the complex Cauchy-Riemann equations it has been shown [1] that the following equation:

$$
\partial_{b} \phi_{2}=\partial_{\bar{b}} \bar{\phi}_{2}
$$

for the constituent $\phi_{2}$ of any $\mathbb{H}$-holomorphic function $\psi(p)=\phi_{1}+\phi_{2} j$ holds. Indeed, according to (1-1) and (1-3), the derivative $\partial_{a} \phi_{1}$ is simultaneously equal to the derivatives $\partial_{\bar{b}} \bar{\phi}_{2}$ and $\partial_{b} \phi_{2}$; hence (4) holds.

The equation (4) can only be satisfied if the constituent $\phi_{2}(a, b)$ has the following general form:

$$
\phi_{2}(a, b)=B\left[(a \bar{a}),(a \bar{a})_{m},(b \bar{b})\right] b,
$$

where the function $B\left[(a \bar{a}),(a \bar{a})_{m},(b \bar{b})\right]$ is dependent on symmetric (in variables $a, \bar{a}$ and $b, \bar{b}$ ) basic forms $a \bar{a}$, $(a \bar{a})_{m}$, and $b \bar{b}$ invariant under complex conjugation. Then $B\left[(a \bar{a}),(a \bar{a})_{m},(b \bar{b})\right]$ is equal to its conjugate $\bar{B}$ and (4) is valid. In accordance with [11] the general expression for the symmetric form $(a \bar{a})_{m}=\overline{(a \bar{a})_{m}}$ can be represented as

$$
\begin{aligned}
(a \bar{a})_{m}= & a^{m} \bar{a}^{0}+a^{(m-1)} \bar{a}^{1}+a^{(m-2)} \bar{a}^{2}+\ldots \\
& +a^{2} \bar{a}^{(m-2)}+a^{1} \bar{a}^{(m-1)}+a^{0} \bar{a}^{m},
\end{aligned}
$$

where $m$ is any non-negative integer. The number of summands in (6) is equal to $(m+1)$. If $m=0$, then we can state that $(a \bar{a})_{0}=a^{0} \bar{a}^{0}=1$.

Since the full derivatives of $\mathbb{H}$-holomorphic functions defined by (2) are $\mathbb{H}$-holomorphic too [11], we can state that the same dependence of $\phi_{2}$ on symmetric invariant forms exists for derivatives of all orders, that is, for $\phi_{2}^{(1)}$, $\phi_{2}^{(2)}$, and so on. The obtained general expressions (5) and (6) now allow us to prove the following assertion, which was discussed earlier in [11] without a proof.

Assertion 1.1 The symmetric (in variables $a, \bar{a}$ ) form of the complex constituents $\phi_{2}^{(k)}, k \geq 1$, of quaternionic $\mathbb{H}$-holomorphic derivatives $\psi_{H}^{(k)}(p)=\phi_{1}^{(k)}+\phi_{2}^{(k)} j$ is a consequence of uniting unsymmetrical constituents $\partial_{a} \phi_{2}^{(k-1)}$ and $\partial_{\bar{a}} \phi_{2}^{(k-1)}$ of the left and right derivatives.

Proof. It suffices to prove this assertion for $k=1$, since every $k^{\prime}$ th derivative is constructed from a previous $\mathbb{H}$-holomorphic $(k-1)^{\prime}$ th derivative. Differentiating $\phi_{2}$, we obtain from (5) the following expressions:

$$
\begin{aligned}
& \partial_{a} \phi_{2}=b\left[\partial_{(a \bar{a})} B\right] \bar{a}+b\left[\partial_{(a \bar{a})_{m}} B\right] \partial_{a}(a \bar{a})_{m}, \\
& \partial_{\bar{a}} \phi_{2}=b\left[\partial_{(a \bar{a})} B\right] a+b\left[\partial_{(a \bar{a})_{m}} B\right] \partial_{\bar{a}}(a \bar{a})_{m} .
\end{aligned}
$$

The derivatives $\partial_{a} \phi_{2}$ and $\partial_{\bar{a}} \phi_{2}$ are the constituents of the left $\left({ }^{\prime} \psi(p)=\partial_{a} \phi_{1}+\partial_{a} \phi_{2} j\right)$ and right $\left(\psi^{\prime}(p)=\right.$ $\left.\partial_{a} \phi_{1}+\partial_{\bar{a}} \phi_{2} j\right)$ derivatives, defined [11] respectively by the left $(1-1,2)$ and right $(1-3,4)$ holomorphicity equations.

Differentiating $(a \bar{a})_{m}$ with respect to $a$ and $\bar{a}$, we get

$$
\begin{aligned}
\partial_{a}(a \bar{a})_{m}= & m a^{(m-1)}+(m-1) a^{(m-2)} \bar{a}+\ldots \\
& +2 a \bar{a}^{(m-2)}+\bar{a}^{(m-1)}, \\
\partial_{\bar{a}}(a \bar{a})_{m}= & a^{(m-1)}+2 a^{(m-2)} \bar{a}+\ldots \\
& +(m-1) a \bar{a}^{(m-2)}+m \bar{a}^{(m-1)} .
\end{aligned}
$$

Combining (2b) for $k=1$, (7), and (8), we obtain

$$
\begin{aligned}
\phi_{2}^{(1)}= & \partial_{a} \phi_{2}+\partial_{\bar{a}} \phi_{2}=b\left[\partial_{(a \bar{a})} B\right](a+\bar{a}) \\
& +b\left[\partial_{(a \bar{a})_{m}} B\right]\left[\partial_{a}(a \bar{a})_{m}+\partial_{\bar{a}}(a \bar{a})_{m}\right] .
\end{aligned}
$$

Finally, substituting (9) and (10) into (11), we have

$$
\phi_{2}^{(1)}=b\left[\partial_{(a \bar{a})} B\right](a \bar{a})_{1}+b(m+1)\left[\partial_{(a \bar{a})_{m}} B\right](a \bar{a})_{m-1} .
$$

Since the derivatives $\partial_{(a \bar{a})} B$ and $\partial_{(a \bar{a})_{m}} B$ are symmetric in $a$ and $\bar{a}$, this expression is symmetric in $a$ and $\bar{a}$ too. Thus the constituent $\phi_{2}^{(k)}$ unites left and right versions of quaternionic analysis, forming its symmetric expression. Q.e.d.

As regards the constituent $\phi_{1}(a, b)$, it can be expressed in the following general form:

$$
\phi_{1}(a, b)=A[a, \bar{a},(b \bar{b})] .
$$

Indeed, the equations (1-2) and (1-4) can be only satisfied if $\phi_{1}(a, b)$ is symmetric in variables $b$ and $\bar{b}$.

Example 1.2 Consider the quaternionic function $p^{4}=(a+b j)^{4}=\phi_{1}+\phi_{2} j$, which is $\mathbb{H}$-holomorphic because it is constructed from the $\mathbb{C}$-holomorphic function $z^{4}$ by replacing $z$ (as a single whole) by $p$ (see Theorem 4.4 in [11]). Straightforward computing yields upon application of (3) the representation of $p^{4}$ in the Cayley-Dickson doubling form, whence further we have 


$$
\begin{gathered}
\phi_{1}=a^{4}-\left(3 a^{2}+2 a \bar{a}+\bar{a}^{2}\right) b \bar{b}+b^{2} \bar{b}^{2}, \\
\phi_{2}=\left[(a \bar{a})_{3}-2(a \bar{a})_{1} b \bar{b}\right] b .
\end{gathered}
$$

The expression for $\phi_{1}$ is symmetric in variables $b$ and $\bar{b}$. The expression for $\phi_{2}$ is symmetric in variables $a$ and $\bar{a}$. The derivatives $\partial_{a} \phi_{2}$ and $\partial_{\bar{a}} \phi_{2}$ are

$$
\begin{aligned}
& \partial_{a} \phi_{2}=\left[\left(3 a^{2}+2 a \bar{a}+\bar{a}^{2}\right)-2 b \bar{b}\right] b, \\
& \partial_{\bar{a}} \phi_{2}=\left[\left(a^{2}+2 a \bar{a}+3 \bar{a}^{2}\right)-2 b \bar{b}\right] b .
\end{aligned}
$$

Summing these, we obtain the following expression:

$$
\phi_{2}^{(1)}=\partial_{a} \phi_{2}+\partial_{\bar{a}} \phi_{2}=4\left[(a \bar{a})_{2}-(a \bar{a})_{0} b \bar{b}\right] b,
$$

which is symmetric in variables $a$ and $\bar{a}$ too.

\section{Relations between Constituents $\phi_{1}, \phi_{2}$}

Recall that we deal with quaternionic holomorphic functions constructed from the corresponding $\mathbb{C}$-holomorphic functions by a direct replacement of a complex variable by a quaternionic in expressions for $\mathbb{C}$-holomorphic functions without change of a functional dependence form. We begin by the theorem:

Theorem 3.1 Let a quaternionic function $\psi_{H}(p)=$ $\phi_{1}(a, b)+\phi_{2}(a, b) j$ be $\mathbb{H}$-holomorphic throughout a connected open set $G_{p} \subset \mathbb{H}$. Assume that $\phi_{1}(a, b)$ and $\phi_{2}(a, b)$ have continuous mixed second order partial derivatives with respect to $a, \bar{a}, b$ and $\bar{b}$, which do not vanish at $p \in G_{p}$. Then, in $G_{p}$ hold true:

$$
\begin{aligned}
& \partial_{a} \phi_{2}=-\partial_{\bar{b}} \phi_{1}, \\
& \partial_{\bar{a}} \phi_{2}=-\partial_{\bar{b}} \bar{\phi}_{1} .
\end{aligned}
$$

Proof. The proof is by reductio ad absurdum. Obviously if the equations (12) and (13) were valid, then we would, by differentiating both sides of the equation (12) with respect to $b$, and by differentiating both sides of the complex conjugate of the equation (13) with respect to $\bar{b}$, have the following equations for mixed derivatives:

$$
\partial_{b a} \phi_{2}=-\partial_{b \bar{b}} \phi_{1}, \partial_{\bar{b} a} \bar{\phi}_{2}=-\partial_{\bar{b} b} \phi_{1}=-\partial_{b \bar{b}} \phi_{1},
$$

where interchanging the order of taking partial derivatives is possible by the condition of the theorem (second-order mixed partial derivatives commute, if they are continuous [10]). (We use here the notation of a type $\partial_{\alpha \beta}$ instead of more usual $\left.\frac{\partial^{2}}{\partial \alpha \partial \beta}\right)$. Subtracting the second equation from the first, we would further have the following relation:

$$
\partial_{b a} \phi_{2}-\partial_{\bar{b} a} \bar{\phi}_{2}=0
$$

Next, assume that any one of equations (12) and (13) at all $p \in G_{p}$ are not valid. Then the expression (14) becomes

$$
\partial_{b a} \phi_{2}-\partial_{\bar{b} a} \bar{\phi}_{2} \neq 0
$$

According to the assumption of the theorem, the second partial derivatives are not to be equal to zero here. By changing the order of taking partial derivatives, we get from (15) the following inequality:

$$
\partial_{a b} \phi_{2} \neq \partial_{a \bar{b}} \bar{\phi}_{2} \text {. }
$$

However this inequality contradicts the true expression

$$
\partial_{a b} \phi_{2}=\partial_{a \bar{b}} \bar{\phi}_{2},
$$

which follows from the equation (4) when differentiating its both sides with respect to $a$. Thus, the assumption (15) is not true; hence the validity of equations (12) and (13) for $\mathbb{H}$-holomorphic functions is proved by reductio ad absurdum. Q.e.d.

We now have proved the validity of the equations (12) and (13) introduced without a proof in [11]. Based on (12) and (13), it is easy to prove one more equation also previously-stated in [11]:

Corollary 3.2 The constituents $\phi_{1}(a, b)$ of the HI-holomorphic functions satisfies the following equation:

$$
\partial_{\bar{a}} \phi_{1}=\partial_{a} \bar{\phi}_{1} \text {. }
$$

Proof. Differentiating both sides of (12) with respect to $\bar{a}$, and both sides of (13) with respect to $a$, we get

$$
\partial_{\bar{a} a} \phi_{2}=-\partial_{\bar{a} \bar{b}} \phi_{1}, \partial_{a \bar{a}} \phi_{2}=-\partial_{a \bar{b}} \bar{\phi}_{1} \text {. }
$$

Taking into consideration that the second partial derivatives commute, and subtracting the second equation from the first, we obtain

$$
\partial_{\bar{b} a} \bar{\phi}_{1}-\partial_{\bar{b} \bar{a}} \phi_{1}=0
$$

and further

$$
\partial_{\bar{b} a} \bar{\phi}=\partial_{\bar{b} \bar{a}} \phi_{1}
$$

If the equation (16) were not valid, then we would have an immediate contradiction to the true equation (17), since by differentiating the inequality $\partial_{\bar{a}} \phi_{1} \neq \partial_{a} \bar{\phi}_{1}$ with respect to $\bar{b}$ we would get $\partial_{\bar{b} a} \bar{\phi}_{1} \neq \partial_{\bar{b} \bar{a}} \phi_{1}$. Hence the equation (16) is valid. Q.e.d.

Putting together equations (4), (12), (13), and (16), we get the following system of equations:

$$
\begin{aligned}
& \text { 1) } \partial_{b} \phi_{2}=\partial_{\bar{b}} \bar{\phi}_{2}, \text { 2) } \partial_{\bar{a}} \phi_{1}=\partial_{a} \overline{\phi_{1}}, \\
& \text { 3) } \partial_{a} \phi_{2}=-\partial_{\bar{b}} \phi_{1} \text {, 4) } \partial_{\bar{a}} \phi_{2}=-\partial_{\bar{b}} \bar{\phi}_{1},
\end{aligned}
$$

which holds if the function $\psi_{H}(p)=\phi_{1}+\phi_{2} j$ is $\mathbb{H}$ holomorphic, that is, $\phi_{1}$ and $\phi_{2}$ satisfy the system (1). Equations $(18-2,3,4)$ are valid if the second mixed partial derivatives don't vanish. These equations enable to deduce various formulae for $\mathbb{H}$-holomorphic derivatives from the basic expressions (2), (2a), and (2b).

To illustrate that such a way generalizes expressions for complex derivatives we consider in detail two quaternionic generalizations:

$$
\begin{aligned}
& \psi_{H}^{(1)}(p)=\left(\partial_{a} \phi_{1}+\partial_{\bar{a}} \phi_{1}\right)+\left(\partial_{a} \phi_{2}+\partial_{\bar{a}} \phi_{2}\right) j, \\
& \psi_{H}^{(1)}(p)=\left(\partial_{a} \phi_{1}+\partial_{a} \bar{\phi}_{1}\right)-\left(\partial_{\bar{b}} \phi_{1}+\partial_{\bar{b}} \bar{\phi}_{1}\right) j,
\end{aligned}
$$

and then the transition from them to complex counterparts.

According to [11], for such a transition it suffices to rule out the dimensions with quaternionic imaginary 
units $i$ and $k$. This can be achieved by the following replacements:

$$
\begin{aligned}
& p=a+b j \in \mathbb{H} \rightarrow \xi=x+z j \in \mathbb{C}, \\
& \psi(p)=\phi_{1}+\phi_{2} j \rightarrow \psi(\xi)=\psi_{1}+\psi_{3} j, \\
& \phi_{1}=\psi_{1}+\psi_{2} i, \bar{\phi}_{1}=\psi_{1}-\psi_{2} i \rightarrow \psi_{1}(x, z), \\
& \phi_{2}=\psi_{3}+\psi_{4} i, \bar{\phi}_{2}=\psi_{3}-\psi_{4} i \rightarrow \psi_{3}(x, z), \\
& \partial_{a}=\frac{1}{2}\left(\partial_{x}-i \partial_{y}\right), \partial^{-}=\frac{1}{2}\left(\partial_{x}+i \partial_{y}\right) \rightarrow \frac{1}{2} \partial_{x}, \\
& \partial_{b}=\frac{1}{2}\left(\partial_{z}-i \partial_{u}\right), \partial^{-}=\frac{1}{2}\left(\partial_{z}+i \partial_{u}\right) \rightarrow \frac{1}{2} \partial_{z} .
\end{aligned}
$$

Performing these in (19) and (20) we get, respectively,

$$
\begin{aligned}
& \psi_{C}^{(1)}(\xi)=\partial_{x} \psi_{1}(x, z)+\partial_{x} \psi_{3}(x, z) j, \\
& \psi_{C}^{(1)}(\xi)=\partial_{x} \psi_{1}(x, z)-\partial_{z} \psi_{1}(x, z) j .
\end{aligned}
$$

These are the expressions for derivatives of complex functions $\psi_{C}(\xi)=\psi_{1}(x, z)+\psi_{3}(x, z) j$ in the complex plane $\xi=x+z j$ with the imaginary unit $j\left(j^{2}=-1\right)$, where $\psi_{C}(\xi)$ satisfies corresponding Cauchy-Riemann's equations

$$
\partial_{x} \psi_{1}=\partial_{z} \psi_{3}, \partial_{x} \psi_{3}=-\partial_{z} \psi_{1} .
$$

It is easy to see also that the complex Cauchy-Riemann equations (21) follow from the quaternionic Cauchy-Riemann equations (1) by the same replacements.

According to the Cayley-Dickson doubling [6] formula: $\gamma=\alpha+\beta j$, where $\alpha$ and $\beta$ are real values when $\gamma$ denotes complex numbers, and $\alpha$ and $\beta$ are complex values when $\gamma$ represents quaternions, while the $j$ remains the same, these replacements represent the transition reverse to the replacement $\xi$ as a single whole by $p$. We see that a representation of initial complex functions in the complex plane $\xi=x+z j$ (with the $j$ instead of the $i$ ) must take place when constructing $\mathbb{H}$-holomorphic functions from complex counterparts.

\section{Particularities of Constructing $\phi_{1}, \phi_{2}$}

As has been said above, we consider the $\mathbb{H}$-holomorphic functions constructed from the $\mathbb{C}$-holomorphic functions by the direct replacement of variables without change of a functional dependence form. The examples considered in [1] are mostly those, in which a complex variable $z$ is replaced as a single whole by a quaternionic variable $p$.

However, if we use additional formulae of complex analysis to represent some $\mathbb{C}$-holomorphic functions, then we must be sure that analogous formulae are valid upon the transition to a quaternion case. Now, we would like to discuss some particularities of constructing the constituents $\phi_{1}$ and $\phi_{2}$, allowing us to be sure that "a functional dependence form" does not change upon such a transition.

1) Computation of constituents $\phi_{1}$ and $\phi_{2}$ when an initial complex counterpart, depending only on $z$ as a single whole, involves the imaginary unit $i$ as a multiplicative factor. For example, the $\mathbb{C}$-holomorphic function $\psi_{C}=i z=z i$, where $i$ and $z=x+y i$ denote, respectively, the imaginary complex unit and a complex variable written in the familiar form. Then, as noted above, the $i$ must be replaced by $j ; z$ by $\xi=x+z j$, and we get the correct initial expression $\psi_{C}=j \xi=\xi j$ to construct the $\mathbb{H}$-holomorphic function. Next the replacement of $\xi$ as a single whole by $p$ gives the $\mathbb{H}$-holomorphic functions $\psi(p)=j p$ and $\psi(p)=p j$.

Consider the function $\psi(p)=j p$. We obtain $\psi(p)=$ $j(a+b j)=-\bar{b}+\bar{a} j$, whence $\phi_{1}=-\bar{b}, \phi_{2}=\bar{a}$ as well as $\bar{\phi}_{1}=-b, \bar{\phi}_{2}=a$. We can see that this function is HI-holomorphic, since the equations (1) hold:

$$
\left\{\begin{array}{l}
\text { 1) } \left.\partial_{a} \phi_{1}=\partial_{\bar{b}} \bar{\phi}_{2}=0,2\right) \partial_{a} \phi_{2}=-\partial_{\bar{b}} \overline{\phi_{1}}=0, \\
\text { 3) } \left.\partial_{a} \phi_{1}=\partial_{b} \phi_{2}=0,4\right) \partial_{\bar{a}} \phi_{2}=-\partial_{\bar{b}} \phi_{1}=1 .
\end{array}\right.
$$

Note that there is no need to use the requirement $a=\bar{a}=x$. The full derivative of this function is in accordance with (19) the following:

$$
(j p)^{(1)}=(0+0)+(0+1) j=j .
$$

This result corresponds to the complex case $(j \xi)^{(1)}=j$. The formula (20) yields the same result.

Consider the function $\psi(p)=p j$. We obtain $\psi(p)=$ $p j=(a+b j) j=-b+a j$, whence $\phi_{1}=-b, \phi_{2}=a$ as well as $\bar{\phi}_{1}=-\bar{b}, \bar{\phi}_{2}=\bar{a}$. This function is $\mathbb{H}$-holomorphic too, since the equations (1) hold:

$$
\left\{\begin{array}{l}
\text { 1) } \left.\partial_{a} \phi_{1}=\partial_{\bar{b}} \bar{\phi}_{2}=0,2\right) \partial_{a} \phi_{2}=-\partial_{\bar{b}} \bar{\phi}_{1}=1, \\
\text { 3) } \left.\partial_{a} \phi_{1}=\partial_{b} \phi_{2}=0,4\right) \partial_{\bar{a}} \phi_{2}=-\partial_{\bar{b}} \phi_{1}=0 .
\end{array}\right.
$$

There is also no need to do the transition $a=\bar{a}=x$, since the derivatives are independent of variables. The derivative of this function in accordance with (19) is as follows:

$$
(p j)^{(1)}=(0+0)+(1+0) j=j .
$$

This corresponds to the complex case too: $(\xi j)^{(1)}=j$.

Consider the function $\psi(p)=j p j$. Since the complex function $\psi_{C}=j \xi j$ is $\mathbb{C}$-holomorphic, the function $\psi(p)=j p j$ must be $\mathbb{H}$-holomorphic too. We have $\psi(p)=j p j=-\bar{a}-\bar{b} j$, whence $\phi_{1}=-\bar{a}, \phi_{2}=-\bar{b}$ as well as $\bar{\phi}_{1}=-a, \bar{\phi}_{2}=-b$. In fact, this function is HI-holomorphic too:

$$
\left\{\begin{array}{l}
\text { 1) } \partial_{a} \phi_{1}=\partial_{\bar{b}} \bar{\phi}_{2}=0, \text { 2) } \partial_{a} \phi_{2}=-\partial_{\bar{b}} \bar{\phi}_{1}=0, \\
\text { 3) } \left.\partial_{a} \phi_{1}=\partial_{b} \phi_{2}=0,4\right) \partial_{\bar{a}} \phi_{2}=-\partial_{\bar{b}} \phi_{1}=0 .
\end{array}\right.
$$

According to (19), the derivative of this function is

$$
(j p j)^{(1)}=(0-1)+(0+0) j=-1 .
$$

It generalizes the complex case too: $(j \xi j)^{(1)}=-1$.

2) Computation of constituents $\phi_{1}$ and $\phi_{2}$ when some additional formulae are used in an initial expression for the $\mathbb{C}$-holomorphic function. For example, by direct replacing $\xi$ by $p$ in the expression for the complex exponential function $\psi(\xi)=e^{\xi}$ we get the quaternionic exponential function $\psi(p)=e^{p}$. To obtain the constituents $\phi_{1}$ and $\phi_{2}$ the next step is to represent this formula in the Cayley-Dickson doubling form $\psi(p)=$ $e^{p}=\phi_{1}+\phi_{2} \cdot j$. One evident way is to use Euler's 
formula $e^{y i}=\cos y+i \sin y$, but first we must establish, in which form this formula is valid in the quaternion area.

The simple replacement of the $i$ by $j$ would be doubtful, because the $i$ is not a multiplicative factor in the function expression depending only on complex variable $z$ as a single whole. We can only use the representation formula for quaternions algebraically equivalent to the complex formula $z=x+y i$. Such a formula is $p=x+v r$, where $v=\sqrt{y^{2}+z^{2}+u^{2}}$ is a real value, $r=\frac{y i+z j+u k}{\sqrt{y^{2}+z^{2}+u^{2}}}$ is a purely imaginary unit quaternion, so its square is -1 .

Then Euler's formula for quaternions is $e^{v r}=\cos v+$ $r \sin v$, and the expression for the quaternionic exponential function $\psi(p)=e^{p}$ is

$$
\begin{aligned}
& e^{p}=e^{(x+v r)}=e^{x} e^{v r} \\
& =e^{x}(\cos v+r \sin v) \\
& =e^{x}\left(\cos v+\frac{y i \sin v}{v}\right)+e^{x} \frac{(z+u i) \sin v}{v} \cdot j,
\end{aligned}
$$

whence $\phi_{1}=e^{x}\left(\cos v+\frac{y i \sin v}{v}\right), \quad \phi_{2}=e^{x} \frac{(z+u i) \sin v}{v}$. The $\mathbb{H}$-holomorphicity of this function is explored in detail in [11]. We shall not dwell on this here.

\section{Expressions for Power Functions}

Taking into account a very important role of computing algorithms in practice, we work out in detail here the expressions for the power functions $p^{n}=(a+b j)^{n}=$ $\phi_{1}(a, b, n)+\phi_{2}(a, b, n) j$, where $n$ is a non-negative integer. We denote further $\phi_{1}(a, b, n)$ and $\phi_{2}(a, b, n)$ simply by $\phi_{1}(n)$ and $\phi_{2}(n)$. For the power functions it is possible to separate variables $a, \bar{a}$ and $b, \bar{b}$ and we can represent $\phi_{1}(n)$ and $\phi_{2}(n)$ by polynomials (sums of monomials) in $b, \bar{b}$ as follows:

$$
\begin{gathered}
\phi_{1}(n)=a^{n}+\sum_{k \geq 0} \phi_{1, k}(n) \\
\phi_{2}(n)=\sum_{k \geq 0} \phi_{2, k}(n),
\end{gathered}
$$

where

$$
\begin{gathered}
\phi_{1, k}(n)=A_{k}(n) \bar{b}^{(k+1)} b^{(k+1)}, \\
\phi_{2, k}(n)=B_{k}(n) \bar{b}^{(k)} b^{(k+1)},
\end{gathered}
$$

and $A_{k}(n)=A_{k}(a, \bar{a}, n), B_{k}(n)=B_{k}\left[(a \bar{a}),(a \bar{a})_{m}, n\right]$.

The term $a^{n}$ in (22) is evident, since $p^{n}$ is the base $p=$ $a+b j$ raised to the power of $n$, hence the variable $a$ too.

Since the sum of exponents of factors included in each monomial $\phi_{1, k}(n)$ and $\phi_{2, k}(n)$ cannot be greater than $n$, and hence the values of $A_{k}(n)$ and $B_{k}(n)$ become zero, respectively if $2 k+2>n$ and $2 k+1>n$, we omit the upper limit of summation over $k$ in (22) and (23) and use the simple symbol $\sum_{k \geq 0}$. It is easy to see that in the above Example $1.2 k=0,1$.

For now we need the multiplication rule

$$
\begin{aligned}
& q_{1} q_{2}=\left(a_{1}+b_{1} j\right)\left(a_{2}+b_{2} j\right) \\
& =\left(a_{1} a_{2}-b_{1} \bar{b}_{2}\right)+\left(a_{1} b_{2}+\bar{a}_{2} b_{1}\right) j,
\end{aligned}
$$

represented in the Cayley-Dickson doubling form [6] for arbitrary quaternions $q_{1}=a_{1}+b_{1} j$ and $q_{2}=a_{2}+b_{2} j$. Setting $q_{1}=p^{n}=\phi_{1}(n)+\phi_{2}(n) j$ and $q_{2}=p=\mathrm{a}+\mathrm{b} j$ we get

$$
p^{(n+1)}=p^{n} p=\phi_{1}(n+1)+\phi_{2}(n+1) j,
$$

where

$$
\begin{aligned}
& \phi_{1}(n+1)=a \phi_{1}(n)-\bar{b} \phi_{2}(n), \\
& \phi_{2}(n+1)=b \phi_{1}(n)+\bar{a} \phi_{2}(n) .
\end{aligned}
$$

Substituting expressions (22) - (25) into the formulae (26) and (27), and grouping the terms with identical exponents of variables $b$ and $\bar{b}$, we get the following expressions:

$$
\begin{aligned}
& \phi_{1}(n+1) \\
& =a^{(n+1)}+\sum_{k \geq 0}\left[a A_{k}(n)-B_{k}(n)\right] \bar{b}^{(k+1)} b^{(k+1)}, \\
& \phi_{2}(n+1)=\left[a^{n}+\bar{a} B_{0}(n)\right] b \\
& +\sum_{k \geq 1}\left[A_{(k-1)}(n)+\bar{a} B_{k}(n)\right] \bar{b}^{(k)} b^{(k+1)} .
\end{aligned}
$$

Representing (28) and (29) in the form similar to (22) and (23), we obtain

$$
\begin{aligned}
& \phi_{1}(n+1)=a^{(n+1)}+\sum_{k \geq 0} A_{k}(n+1) \bar{b}^{(k+1)} b^{(k+1)}, \\
& \phi_{2}(n+1)=\sum_{k \geq 1} B_{k}(n+1) b^{-(k)} b^{(k+1)},
\end{aligned}
$$

where

$$
\begin{gathered}
A_{k}(n+1)=a A_{k}(n)-B_{k}(n), k \geq 0, \\
B_{k}(n+1)=A_{(k-1)}(n)+\bar{a} B_{k}(n), k \geq 1, \\
B_{0}(n+1)=a^{n}+\bar{a} B_{0}(n) .
\end{gathered}
$$

Since in accordance with the associativity of quaternion multiplication the equality $p^{(n+1)}=p p^{n}$ holds too, we get

$$
\begin{aligned}
& \phi_{1}(n+1)=a \phi_{1}(n)-b \bar{\phi}_{2}(n), \\
& \phi_{2}(n+1)=b \bar{\phi}_{1}(n)+a \phi_{2}(n),
\end{aligned}
$$

whence, arguing as above, we deduce the expressions

$$
\begin{gathered}
A_{k}(n+1)=a A_{k}(n)-\bar{B}_{k}(n), k \geq 0, \\
B_{k}(n+1)=\bar{A}_{(k-1)}(n)+a B_{k}(n), k \geq 1, \\
B_{0}(n+1)=\bar{a}^{n}+a B_{0}(n),
\end{gathered}
$$

equivalent to (30) - (32).

Theorem 5.1 Let a quaternion power function $p^{n}=$ $\phi_{1}(n)+\phi_{2}(n) j$, where $n$ is any non-negative integer and $\phi_{1}(n)$ and $\phi_{2}(n)$ are differentiable with respect to $a, \bar{a}, b$, 
$\bar{b}$, be represented by polynomials in $b, \bar{b}$. Then the factors $A_{k}(n)$ and $B_{k}(n)$ satisfy the following relations:

$$
\begin{gathered}
B_{0}(n)=(a \bar{a})_{(n-1)}, n \geq 1, \\
A_{k}(n)=-(k+1)^{-1} \partial_{a} B_{k}(n), k \geq 0, \\
B_{k}(n)=k^{-1} \partial_{a} \bar{A}_{(k-1)}(n), k \geq 1, \\
B_{k}(n)=-k^{-2} \partial_{a \bar{a}} B_{(k-1)}(n), k \geq 1 .
\end{gathered}
$$

Proof. The proof will be divided into 2 steps.

Step 1. First we prove the formulae (36), and then (37) for $k=0$ :

$$
A_{0}(n)=-\partial_{a} B_{0}(n), n \geq 1 .
$$

Relabeling $(n+1)$ as $n$ in the recursive relation (32), we get

$$
B_{0}(n)=a^{(n-1)}+\bar{a} B_{0}(n-1), n \geq 1 .
$$

To eliminate recursion from the function $B_{0}(n)$ we must use (41) repeatedly until obtaining an explicit closeform expression, that is, until obtaining the obvious base case:

$$
B_{0}(1)=1 .
$$

(It is easy to see that from $p^{1}=\mathrm{a}+\mathrm{b} j=\phi_{1}(1)+\phi_{2}(1) j$ we have $\phi_{1}(1)=a$ and $A_{0}(1)=0$ as well as $\phi_{2}(1)=b$ and $B_{0}(1)=1$.) Using the relation (41) two times, we have

$$
\begin{aligned}
B_{0}(n) & =a^{(n-1)}+\bar{a}\left[a^{(n-2)}+\bar{a} B_{0}(n-2)\right] \\
& =a^{(n-1)}+\bar{a} a^{(n-2)}+\bar{a}^{2} B_{0}(n-2) .
\end{aligned}
$$

Further, using the relation (41) $\lambda$ times, we obtain the following recursive relation:

$$
\begin{aligned}
B_{0}(n)= & a^{(n-1)}+\bar{a} a^{(n-2)}+\bar{a}^{2} a^{(n-3)}+\ldots \\
& +\bar{a}^{(\lambda-1)} a^{(n-\lambda)}+\bar{a}^{\lambda} B_{0}(n-\lambda) .
\end{aligned}
$$

Taking into account (42), we see that the iteration must be terminated after $\lambda=(\mathrm{n}-1)$ steps when $n-\lambda=1$. Thus we have the following explicit close-form expression:

$$
\begin{aligned}
B_{0}(n)= & a^{(n-1)}+a^{(n-2)} \bar{a}+a^{(n-3)} \bar{a}^{2}+\ldots \\
& +a^{2} \bar{a}^{(n-3)}+a \bar{a}^{(n-2)}+\bar{a}^{(n-1)} .
\end{aligned}
$$

Comparing this expression with (6), we finally get:

$$
B_{0}(n)=(a \bar{a})_{(n-1)} .
$$

The statement (36) of Theorem 5.1 is proved.

The formula (6) can be also represented as

$$
(a \bar{a})_{m}=a(a \bar{a})_{(m-1)}+\bar{a}^{m},
$$

then, differentiating it with respect to $a$, we get

$$
\partial_{a}(a \bar{a})_{m}=(a \bar{a})_{(m-1)}+a \partial_{a}(a \bar{a})_{(m-1)} .
$$

Combining (36) and (43) we obtain further the recursive formula for $\partial_{a} B_{0}(n)$ :

$$
\partial_{a} B_{0}(n)=\partial_{a}(a \bar{a})_{(n-1)}=(a \bar{a})_{(n-2)}+a \partial_{a}(a \bar{a})_{(n-2)} .
$$

Substituting (43) into this formula one more time, that is, using (43) in the expression for $\partial_{a} B_{0}(n)$ two times, we get the following expression:

$$
\partial_{a} B_{0}(n)=(a \bar{a})_{(n-2)}+a(a \bar{a})_{(n-3)}+a^{2} \partial_{a}(a \bar{a})_{(n-3)} .
$$

Using the relation (43) $\lambda$ times, we obtain:

$$
\begin{aligned}
\partial_{a} B_{0}(n)= & (a \bar{a})_{(n-2)}+a(a \bar{a})_{(n-3)}+a^{2}(a \bar{a})_{(n-4)}+\ldots \\
& +a^{(\lambda-1)}(a \bar{a})_{(n-\lambda-1)}+a^{(\lambda)} \partial_{a}(a \bar{a})_{(n-\lambda-1)} .
\end{aligned}
$$

The iteration terminates after $\lambda=n-1$ steps when $n-\lambda-1=0,(a \bar{a})_{0}=1, \partial_{a}(a \bar{a})_{0}=0$. Given this, we obtain the following expression for the derivative:

$$
\begin{aligned}
\partial_{a} B_{0}(n)= & (a \bar{a})_{(n-2)}+a(a \bar{a})_{(n-3)}+a^{2}(a \bar{a})_{(n-4)}+\ldots \\
& +a^{(n-4)}(a \bar{a})_{2}+a^{(n-3)}(a \bar{a})_{1}+a^{(n-2)}(a \bar{a})_{0} .
\end{aligned}
$$

Finally, making use of (36), we have

$$
\begin{gathered}
\partial_{a} B_{0}(n)=B_{0}(n-1)+a B_{0}(n-2)+a^{2} B_{0}(n-3)+\ldots \\
\quad+a^{(n-4)} B_{0}(3)+a^{(n-3)} B_{0}(2)+a^{(n-2)} B_{0}(1) .
\end{gathered}
$$

It remains to get an analogous expression for $A_{0}(n)$. We use the formula (30) for $k=0$, relabelling $n$ as $(n-1)$ :

$$
A_{0}(n)=a A_{0}(n-1)-B_{0}(n-1), n \geq 1,
$$

Using (45) $\lambda$ times (with respect to $A_{0}(n)$ ) like above, we get the following recursive formula for $A_{0}(n)$ :

$$
\begin{aligned}
A_{0}(n)= & a^{\lambda} A_{0}(n-\lambda)-a^{(\lambda-1)} B_{0}(n-\lambda) \\
& -a^{(\lambda-2)} B_{0}(n-\lambda+1)-\ldots-a^{2} B_{0}(n-3) \\
& -a B_{0}(n-2)-B_{0}(n-1) .
\end{aligned}
$$

The iteration terminates after $\lambda=n-1$ steps, since $n-\lambda$ becomes 1 , and we achieve the base case of the recurrence: $A_{0}(1)=0$ and $B_{0}(1)=1$. Finally, we get for $A_{0}(n)$ :

$$
\begin{aligned}
A_{0}(n)= & -a^{(n-2)} B_{0}(1)-a^{(n-3)} B_{0}(2) \\
& -a^{(n-4)} B_{0}(3)-\ldots-a^{2} B_{0}(n-3) \\
& -a B_{0}(n-2)-B_{0}(n-1) .
\end{aligned}
$$

Comparing (46) with (44), we immediately prove that (40), that is, (37) for $k=0$ is valid.

Step 2. We prove the formulae (37) and (38) for $k \geq 1$ and $n \geq 1$ by using mathematical induction.

The base case $n=1, p=a+b j$, whence, given (22) (25), we have the sequences $A_{k}(1): A_{0}(1)=A_{1}(1)=$ $A_{2}(1)=\ldots=0$ and $B_{k}(1): B_{0}(1)=1, B_{1}(1)=B_{2}(1)=$ $\ldots=0$. Applying (37) to elements of $B_{k}(1)$, we obtain $A_{1}(1)=-2^{-1} \partial_{a} B_{1}(1)=0$, and so on, which coincide 
with the corresponding elements of $A_{k}(1)$. Applying (38) to elements of $A_{k}(1)$, we obtain $B_{1}(1)=\partial_{a} \bar{A}_{0}(1)=0$, $B_{2}(1)=2^{-1} \partial_{a} \bar{A}_{1}(1)=0$ and so on, which coincide with the corresponding elements of $B_{k}(1)$. The formulae (37) and (38) are true in this case.

The base case $n=2, p^{2}=(a+b j)(a+b j)$. By direct multiplication and using (3), we get in the Cayley-Dickson doubling form $p^{2}=a^{2}-b \bar{b}+(a \bar{a})_{1} b j$, whence $\phi_{1}(2)=$ $a^{2}-b \bar{b}$ and $\phi_{2}(2)=(a \bar{a})_{1} b$. Given (22) - (25), we have the sequences $A_{k}(2): A_{0}(2)=-1, A_{1}(2)=A_{2}(2)=$ $\ldots=0$ and $B_{k}(2): B_{0}(2)=(a \bar{a})_{1}, B_{1}(2)=B_{2}(2)=\ldots=$ 0 . Applying (37) to elements of $B_{\mathrm{k}}(2)$, we obtain $A_{0}(2)=$ $-\partial_{a} B_{0}(2)=-1, A_{1}(2)=-2^{-1} \partial_{a} B_{1}(2)=0$, and so on, which coincide with the corresponding elements of $A_{k}$ (2). Applying (38) to elements of $A_{k}(2)$, we obtain $B_{1}(2)=\partial_{a} \bar{A}_{0}(2)=0, \quad B_{2}(2)=2^{-1} \partial_{a} \bar{A}_{1}(2)=0$ and so on, which coincide with the corresponding elements of $B_{k}(2)$. The formulae (37) and (38) are true in this case too. We see that the values of $A_{k}(n)$ and $B_{k}(n)$ become zero in both base cases automatically when values of $k$ increasing as noted above.

The inductive step. Assuming that the equations (37) and (38) are valid for any $n \geq 1$, we will prove the validity of these equations for $n+1$ :

$$
\begin{gathered}
A_{k}(n+1)=-(k+1)^{-1} \partial_{a} B_{k}(n+1), k \geq 1, \\
B_{k}(n+1)=k^{-1} \partial_{a} \bar{A}_{(k-1)}(n+1), k \geq 1 .
\end{gathered}
$$

Substituting (30) and (34) into (47), we obtain the following expression:

$$
\begin{aligned}
& (k+1)\left[a A_{k}(n)-B_{k}(n)\right] \\
& =-\partial_{a}\left[\bar{A}_{(k-1)}(n)+a B_{k}(n)\right], k \geq 1 .
\end{aligned}
$$

Performing the differentiation on the right-hand side of this expression and simplifying, we get as follows:

$$
\begin{aligned}
& a(k+1) A_{k}(n)-k B_{k}(n)+a \partial_{a} B_{k}(n) \\
& =-\partial_{a} \bar{A}_{(k-1)}(n), k \geq 1 .
\end{aligned}
$$

Using (37), we now come to the expression

$$
B_{k}(n)=k^{-1} \partial_{a} \bar{A}_{(k-1)}(n), k \geq 1,
$$

that is valid by the hypothesis of induction (38). Thus, the relation (47) is valid if the relations (37) and (38) are supposed to be valid. It remains to prove the validity of the expression (48) if the validity of the expression (37) and (38) is supposed.

By complex conjugating (33), and relabelling $k$ as $k-1$, we get

$$
\bar{A}_{(k-1)}(n+1)=\bar{a} \bar{A}_{(k-1)}(n)-B_{(k-1)}(n), k \geq 1 .
$$
have

Differentiating this expression with respect to $a$, we

$$
\partial_{a} \bar{A}_{(k-1)}(n+1)=\bar{a} \partial_{a} \bar{A}_{(k-1)}(n)-\partial_{a} B_{(k-1)}(n), k \geq 1 .
$$

Further, multiplying (31) by $k$ yields

$$
k B_{k}(n+1)=k A_{(k-1)}(n)+k \bar{a} B_{k}(n), k \geq 1 .
$$

Given the last two results, the expression (48) becomes

$$
\begin{aligned}
& k A_{(k-1)}(n)+k \bar{a} B_{k}(n) \\
& =\bar{a} \partial_{a} \bar{A}_{(k-1)}(n)-\partial_{a} B_{(k-1)}(n), k \geq 1 .
\end{aligned}
$$

Relabeling $k$ as $k-1$ in the formula (37), we have

$$
A_{(k-1)}(n)=-k^{-1} \partial_{a} B_{(k-1)}(n), k \geq 1,
$$

Finally, substituting (50) into (49), we get the following expression:

$$
B_{k}(n)=k^{-1} \partial_{a} \bar{A}_{(k-1)}(n), k \geq 1,
$$

which is valid by the hypothesis of induction (38). Thus, the validity of the expression (48) is also proved if the validity of (37) and (38) is supposed. In sum, we have proved by induction on $n$ that the statements (37) and (38) of Theorem 5.1 are valid.

The validity of the statement (39) follows from the relations (37) and (38). Indeed, substituting the conjugate of the expression (50) into (38) and taking into account that

$$
B_{(k-1)}(n)=B_{(k-1)}\left[(a \bar{a}),(a \bar{a})_{m}, n\right]=\bar{B}_{(k-1)}(n),
$$

we obtain the expression (39). Thus, the statement (39) of Theorem 5.1 is proved too. This completes the proof of theorem 5.1 in whole. Q.e.d.

By starting with the expression (36), the recursive relation (39) allows us to compute step by step the factors $B_{k}(n)$ for all $k \geq 1$. The formulae (37) and (38) represent the relations between the factors $A_{k}(n)$ and $B_{k}(n)$, hence between the constituents $\phi_{1}(n)$ and $\phi_{2}(n)$. It is not difficult to show that, computing the derivatives of functions represented by (22), (23) to be used in (12) and (13), and combining the obtained results with (37), we get the equations (12) and (13) for power functions.

To illustrate how the relations (37) - (39) work we continue the consideration of Example 1.2. Recall that the function $p^{4}=(a+b j)^{4}$ represented in the CayleyDickson doubling form as $p^{4}=\phi_{1}(4)+\phi_{2}(4) j$ has

$$
\begin{gathered}
\phi_{1}(4)=a^{4}-\left(3 a^{2}+2 a \bar{a}+\bar{a}^{2}\right) b \bar{b}+b^{2} \bar{b}^{2}, \\
\phi_{2}(4)=\left[(a \bar{a})_{3}-2(a \bar{a})_{1} b b\right] b .
\end{gathered}
$$

Given (22) - (25), we obtain from these expressions the sequences $A_{k}(4): A_{0}(4)=-\left(3 a^{2}+2 a \bar{a}+\bar{a}^{2}\right), A_{1}(4)=$ $1, A_{2}(4)=A_{3}(4)=\cdots=0$, and $B_{k}(4): B_{0}(4)=(a \bar{a})_{3}$, $B_{1}(4)=-2(a \bar{a})_{1}, B_{2}(4)=B_{3}(4)=\cdots=0$. Applying (37) to the corresponding elements of the sequence $B_{k}(4)$, we obtain the expressions $A_{0}(4)=-\partial_{a} B_{0}(4)=-\partial_{a}(a \bar{a})_{3}=$ $-\left(3 a^{2}+2 a \bar{a}+\bar{a}^{2}\right), A_{1}(4)=\partial_{a}(a \bar{a})_{1}=1, A_{2}(4)=$ $A_{3}(4)=\cdots=0$. The formula (37) gives the values of $A_{k}(4)$, coinciding with the elements of the above sequence $A_{k}(4)$.

On the other hand, applying the formula (38) to the corresponding elements of the sequence $A_{k}(4)$, we get

$$
\begin{aligned}
& B_{1}(4)=-1^{-1} \partial_{a}\left(3 \bar{a}^{2}+2 a \bar{a}+a^{2}\right) \\
& =-(2 \bar{a}+2 a)=-2(a \bar{a})_{1},
\end{aligned}
$$




$$
B_{2}(4)=2^{-1} \partial_{a}(1)=0, B_{3}(4)=B_{4}(4)=\ldots=0 .
$$

The formula (38) gives the values, coinciding with values of elements of the above sequence $B_{k}(4)$.

The initial value $B_{0}(4)=(a \bar{a})_{3}$ satisfies (36). The formula (39) provides consistently the following $B_{k}(n)$ :

$$
\begin{aligned}
B_{1}(4) & =-1^{-1} \partial_{a \bar{a}}(a \bar{a})_{3} \\
& =-\partial_{a}\left(a^{2}+2 a \bar{a}+3 \bar{a}^{2}\right)=-2(a \bar{a})_{1}, \\
B_{2}(4) & =-4^{-1} \partial_{a \bar{a}} B_{1}(4)=2^{-1} \partial_{a \bar{a}}(a \bar{a})_{1}=0, \\
B_{3}(4) & =B_{4}(4)=\ldots=0,
\end{aligned}
$$

coinciding with the values of the initial sequence $B_{k}(4)$. This example demonstrates the validity of relations (36)-(39).

\section{Acknowledgements}

I am indebted to Irina Levina for the continued support and Ephraim Gurevich for useful discussions.

\section{References}

[1] De Leo, S.,Rotelli, P.P., "Quaternionic analyticity", Applied Mathematics Letters, 16, 1077-1081, 2003.

[2] Dzagnidze, O., On the differentiability of quaternion functions, Mar. 2012. [arXiv: math.CV] Available: arxiv.org/abs/1203.5619.
[3] Frenkel, I., Libine, M., Quaternionic analysis, representation theory and physics, July 2011. [arXiv: math.CV] Available: arxiv.org/abs/0711.2699v5

[4] Gentili, G., Struppa, D. C., "A new theory of regular functions of a quaternionic variable," Advances in Mathematics, 216, 279-301, 2007.

[5] Grigor'ev, Yu., "Regular quaternionic functions and their applications," in 20th International Conference on the Application of Computer Science and Mathematics in Architecture and Civil Engineering, Weimar, Germany, 20-22 July 2015

[6] Gürlebeck, K., Habetta, K., Sprößig, W., Funktionentheorie in der Ebene und im Raum, Birkhäuser Verlag, Basel - Boston - Berlin, 2006.

[7] Kantor, I. L., Solodovnikov, A. S., Hypercomplex Numbers. An Elementary Introduction to Algebras, Springer-Verlag, New YorkBerlin-Heidelberg-London-Paris-Tokyo, 1989

[8] Królikowski, W., "New property of quaternionic Fueter functions," Journal of Applied Mathematics and Computational Mechanics, 12(2), 29-37, 2013.

[9] Leutwiler, H., Zeilinger, P., "On Quaternionic Analysis and its Modifications", Computational Methods and Function Theory, 4 (1), 159-182, 2004.

[10] J. H. Mathews, R. W. Howell, Complex Analysis for Mathematics and Engineering, 3rd ed., Jones and Bartlett Publishers, BostonToronto-London-Singapore, 1997.

[11] Parfenov, M., Adequate quaternionic generalization of complex differentiability, [viXra: Functions and Analysis] Aug 2016. Available: vixra.org/abs/1609.0006

[12] Sudbery, A., "Quaternionic analysis," Math. Proc. Camb. Phil. Soc., 85, 199-225, 1979.

[13] Xu, D., Jahanchahi, C., Took, C.C., Mandic D.P., "Enabling quaternion derivatives: the generalized HR calculus," R. Soc. open sci., 2: 150255, Aug.2015 Available: rsos.royalsocietypublishing.org/content/2/8/150255. 